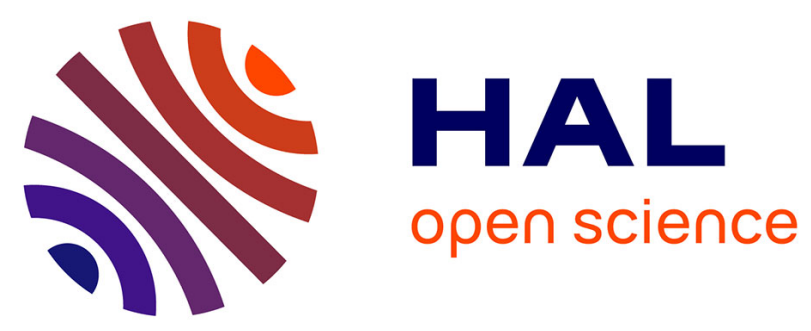

\title{
A boundary value problem for conjugate conductivity equations
}

\author{
Slah Chaabi, Stéphane Rigat, Franck Wielonsky
}

\section{To cite this version:}

Slah Chaabi, Stéphane Rigat, Franck Wielonsky. A boundary value problem for conjugate conductivity equations. Studies in Applied Mathematics, 2016, 137 (3), pp.328-355. 10.1111/sapm.12124 . hal01146281

\section{HAL Id: hal-01146281 \\ https://hal.science/hal-01146281}

Submitted on 28 Apr 2015

HAL is a multi-disciplinary open access archive for the deposit and dissemination of scientific research documents, whether they are published or not. The documents may come from teaching and research institutions in France or abroad, or from public or private research centers.
L'archive ouverte pluridisciplinaire HAL, est destinée au dépôt et à la diffusion de documents scientifiques de niveau recherche, publiés ou non, émanant des établissements d'enseignement et de recherche français ou étrangers, des laboratoires publics ou privés.

\section{다(1)(2)}

Distributed under a Creative Commons Attribution - ShareAlike| 4.0 International 


\title{
A boundary value problem for conjugate conductivity equations
}

\author{
S. Chaabi, S. Rigat and F. Wielonsky
}

April 27, 2015

\begin{abstract}
We give explicit integral formulas for the solutions of planar conjugate conductivity equations in a circular domain of the right half-plane with conductivity $\sigma(x, y)=x^{p}, p \in \mathbb{Z}^{*}$. The representations are obtained via a Riemann-Hilbert problem on the complex plane when $p$ is even and on a two-sheeted Riemann surface when $p$ is odd. They involve the Dirichlet and Neumann data on the boundary of the domain. We also show how to make the conversion from one type of conditions to the other by using the so-called global relation. The method used to derive our integral representations could be applied in any bounded simply-connected domain of the right half-plane with a smooth boundary.
\end{abstract}

Keywords: Boundary value problem, Riemann-Hilbert problem, Lax pair

MCS: 35J25, 35Q15, 35J15

\section{Introduction and main results}

We seek explicit integral expressions for real-valued solutions of the Neumann/Dirichlet problem for the following two conductivity equations, considered in a bounded simply connected domain $\Omega$ of the right half-plane $\mathbb{H}=\left\{(x, y) \in \mathbb{R}^{2}, x>0\right\}$,

$$
\operatorname{Div}(\sigma \nabla u)=0, \quad \operatorname{Div}\left(\frac{1}{\sigma} \nabla v\right)=0,
$$

for the particular case of conductivity $\sigma(x, y)=x^{p}, p \in \mathbb{Z}$. For a general conductivity $\sigma(x, y)>0, x \in \mathbb{H}$, the two equations are conjugate in the following sense. If the function $u$ satisfies the first equation in (1.1) then the differential form $-\sigma \partial_{y} u d x+\sigma \partial_{x} u d y$ is closed, so by applying Poincaré theorem in the simply connected $\Omega$, there exists a function $v$ such that

$$
\partial_{x} v=-\sigma \partial_{y} u, \quad \partial_{y} v=\sigma \partial_{x} u .
$$

Then, $v$ solves the second equation in (1.1). Conversely, for such a $v$, the differential form $\sigma^{-1} \partial_{y} v d x-\sigma^{-1} \partial_{x} v d y$ is closed and there exists a function $u$ that satisfies (1.2) and the first equation in (1.1). Moreover, letting

$$
\nu=(1-\sigma) /(1+\sigma) \in \mathbb{R},
$$


we have that (1.2) is equivalent to the so-called conjugate Beltrami equation,

$$
\bar{\partial} f=\nu \overline{\partial f}
$$

for the complex-valued function $f=u+i v$ in $\Omega$. This equation differs substantially from the classical and extensively studied Beltrami equation $\bar{\partial} f=\nu \partial f$. The link between the conductivity equations (1.1) and (1.3) was used in [3] to investigate the Dirichlet to Neumann map. For a detailed study of the conjugate Beltrami equation (1.3) see e.g. [4].

When $\sigma=1$, the equations in (1.1) reduce to the Laplace equation and (1.2) to the Cauchy-Riemann equations. When $\sigma$ differs from 1, we may speak, by analogy, of a $\sigma$ harmonic function $u$ and a $\nu$-holomorphic function $f$. For the conductivity $\sigma(x, y)=x^{p}$, $p \in \mathbb{N}^{*}$, the equations in (1.1) rewrite as

$$
\begin{aligned}
& \Delta u+\frac{p}{x} \partial_{x} u=0 \\
& \Delta v-\frac{p}{x} \partial_{x} v=0 .
\end{aligned}
$$

These equations are particular case of the general elliptic equation

$$
\Delta u+\frac{\alpha}{x} \partial_{x} u=0, \quad \alpha \in \mathbb{R}
$$

which is the topic of the so-called generalized axially symmetric potential theory (GASPT), see [29]. The name comes from the relation with the Laplacian in spaces of higher dimensions. For instance, let $(r, \varphi, z)$ denotes the usual cylindrical coordinates in $\mathbb{R}^{3}, \Omega$ be a domain in the $(x, z)$-plane, and $\Omega^{\prime} \subset \mathbb{R}^{3}$ be the domain obtained by rotation of $\Omega$ about the $z$-axis. Then, $u(r, z)$ is a solution of $\Delta u(r, z)+r^{-1} \partial_{r} u(r, z)=0$ in $\Omega$ if and only if $u(r, z)$ is harmonic in $\Omega^{\prime}$. Actually, a similar relation holds for the more general equation (1.4) when $p \in \mathbb{N}^{*}$. Indeed, if $r=\left(x_{1}^{2}+\cdots+x_{p+1}^{2}\right)^{1 / 2}$ and $U\left(x_{1}, \ldots, x_{p+2}\right)=u\left(r, x_{p+2}\right)$ then one checks easily that

$$
\Delta U\left(x_{1}, \ldots, x_{p+2}\right)=\Delta u\left(r, x_{p+2}\right)+\frac{p}{r} \partial_{r} u .
$$

When considering a domain $\Omega$ with some simple geometry, this link with the Laplacian allows one to get explicit bases of solutions via the method of separation of variables. For instance, toroidal harmonics (i.e. Legendre functions with half-integer degrees, see e.g. [19]) give a complete set of solutions to (1.6) when $\alpha= \pm 1$, cf. $[2,22,26]$. The fact that generalized Legendre functions can be used to solve (1.6) for complex values of $\alpha$ appears in $[6,7]$.

The GASPT theory has been investigated by many authors, among whom Weinstein [28-31], Vekua [27], Gilbert [12-15], Henrici [16-18], Mackie [21], Ranger [23]. Equation (1.6) is related to a variety of problems in physics. In particular, when $p=1$, equation (1.4) can be interpreted as the linearized version of the Ernst equation in the case of a static spacetime. In [20], this equation was studied in the unbounded domain consisting of the exterior of the real segment $\left(0, \rho_{0}\right)$ in the right half-plane $\mathbb{H}$ and was related to the relativistic gravitational field produced by a rotating disk of matter. Equation (1.5) with 
$p=1$ is related to the behavior of plasma in a tokamak. The goal of this device, which has a toroidal geometry, is to control location of the plasma in its chamber by applying magnetic fields on the boundary. From an assumption of axial symmetry, the problem is reduced to a plane section. Then, it follows from the Grad-Shafranov equation, a secondorder elliptic nonlinear partial differential equation, see $[5,24]$, that the magnetic flux in the vacuum between the plasma and the circular boundary of the chamber satisfies the homogeneous equation (1.5). Note, that in this instance, the conductivity equation (1.5) takes place in an annular domain, that is a doubly connected domain.

Another interesting feature of equation (1.6) is that it satisfies recurrence and symmetry relations with respect to the coefficient $\alpha$. Namely, denoting by $\left(E_{\alpha}\right)$ the equation (1.6), the following holds:

$$
\begin{aligned}
& u(x, y) \text { solves }\left(E_{\alpha}\right) \text { if and only if } x^{-1} \partial_{x} u(x, y) \text { solves }\left(E_{\alpha+2}\right), \\
& u(x, y) \text { solves }\left(E_{\alpha}\right) \text { if and only if } x^{\alpha-1} u(x, y) \text { solves }\left(E_{2-\alpha}\right),
\end{aligned}
$$

see e.g. [30]. Finally, note that, since equation (1.6) is elliptic, we know, by general results, see e.g. [11], that the Dirichlet problem with datas on the boundary $\partial \Omega$ of the domain has a unique solution, which is $C^{\infty}$ in $\Omega$.

In the present paper, we will stick, for simplicity, to the case of a domain $\Omega$ being the open disk $\mathcal{D}_{a}=D(a, 1)$, lying in the right half-plane $\mathbb{H}$, with $a>1$ a point on the positive real axis. As briefly explained at the end of Section 3, the approach used to derive our integral representations could be adapted to a general Jordan domain $\Omega$ in $\mathbb{H}$ bounded by a smooth curve.

The first results of our study are the following Theorems 1.1 and 1.3, which give explicit integral representations for the solutions of equation (1.6) when $\alpha$ is an even and odd negative integer respectively. The method of proof follows the general scheme of the socalled unified transform method, see [8]. It uses a Lax pair, from which one may define a function $\phi(z, k)$ depending on a spectral parameter $k$, and which can be characterized as the solution of a specific singular Riemann-Hilbert problem in the $k$-plane. Solving this Riemann-Hilbert problem leads to the seeked integral representations.

In the sequel, for $z$ inside the disk $\mathcal{D}_{a}$, we denote by $z_{r}$ the point on the circle $\mathcal{C}_{a}=$ $C(a, 1)$ which has the same imaginary part as $z$ and lies to its right.

Theorem 1.1. Let $u$ be a solution of the equation

$$
\Delta u+\alpha x^{-1} \partial_{x} u=0, \quad \alpha=-2 m, \quad m \in \mathbb{N},
$$

in the domain $\mathcal{D}_{a}$ with smooth tangential and (outer) normal derivatives $u_{t}$ and $u_{n}$ on the boundary $\mathcal{C}_{a}$. Then $u$ admits the integral representation

$$
u(z)=-\frac{1}{\pi} \operatorname{Im} \int_{\left(z, z_{r}\right)}((k-z)(k+\bar{z}))^{m} J(z, k) d k+2 \operatorname{Re}\left(a_{r}\right)+u\left(z_{r}\right), \quad z \in \mathcal{D}_{a},
$$

where integration is on the segment $\left(z, z_{r}\right)$, and the quantity $a_{r}$ can be explicitly computed in terms of the tangential derivatives along $\mathcal{C}_{a}$ of $u_{t}$ and $u_{n}$, up to order $m-1$, at $z_{r}$. The function $J(z, k)$ is given by

$$
J(z, k)=-\int_{\mathcal{C}_{a}} W\left(z^{\prime}, k\right)
$$


where $W(z, k)$ is the differential form

$$
\begin{aligned}
W(z, k) & =((k-z)(k+\bar{z}))^{-m-1}\left((k+\bar{z}) u_{z}(z) d z+(k-z) u_{\bar{z}}(z) d \bar{z}\right) \\
& =((k-z)(k+\bar{z}))^{-m-1}\left((k-i y) u_{t}(z)+i x u_{n}(z)\right) d s
\end{aligned}
$$

with $z=x+i y$ and $d s$ the length element on $\mathcal{C}_{a}$.

Remark 1.2. In the particular case $\alpha=m=0$, equation (1.6) is Laplace equation and the solutions $u$ are simply the functions harmonic in $\mathcal{D}_{a}$. In this case, the expression in the right-hand side of (1.9) simplifies to

$$
\begin{aligned}
& \frac{1}{\pi} \operatorname{Im} \int_{\left(z, z_{r}\right)} \int_{\mathcal{C}_{a}} W\left(z^{\prime}, k\right) d k+u\left(z_{r}\right)=\frac{1}{\pi} \operatorname{Im} \int_{\left(z, z_{r}\right)} \int_{\mathcal{C}_{a}}\left(\frac{u_{z}(z)}{k-z} d z+\frac{u_{\bar{z}}(z)}{k+\bar{z}} d \bar{z}\right) d k+u\left(z_{r}\right) \\
& =\frac{1}{\pi} \operatorname{Im} \int_{\left(z, z_{r}\right)}-2 i \pi u_{z}(k) d k+u\left(z_{r}\right)=-\int_{\left(z, z_{r}\right)} u_{x}(x) d x+u\left(z_{r}\right)
\end{aligned}
$$

which is indeed $u(z)$. Note that in the second equality we have applied Cauchy formula to the analytic function $u_{z}(z)$.

Theorem 1.3. Let $u$ be a solution of the equation

$$
\Delta u+\alpha x^{-1} \partial_{x} u=0, \quad \alpha=-2 m+1, \quad m \in \mathbb{N},
$$

in the domain $\mathcal{D}_{a}$ with smooth tangential and (outer) normal derivatives $u_{t}$ and $u_{n}$ on the boundary $\mathcal{C}_{a}$. Then $u$ admits the integral representation

$$
u(z)=-\frac{1}{2 \pi} \operatorname{Im} \int_{\mathcal{C}_{a}} \frac{\left(\left(k-z_{r}\right)\left(k+\bar{z}_{r}\right)\right)^{m} J(z, k)}{\sqrt{(k-z)(k+\bar{z})}} d k+u\left(z_{r}\right), \quad z \in \mathcal{D}_{a},
$$

where integration is on the circle $\mathcal{C}_{a}$, oriented counter-clockwise. The square root in the denominator has a branch cut along the segment $(-\bar{z}, z)$. Integration starts at $z_{r}$ where the square root is taken to be positive, and its determination is chosen so that it remains continuous along the path of integration. The function $J(z, k)$ is explicitly given in terms of the tangential and normal derivatives $u_{t}$ and $u_{n}$ and their derivatives of order up to $m-1$ along the boundary $\mathcal{C}_{a}$. It can be written as a sum,

$$
J(z, k)=J^{0}\left(z_{r}, k\right)+\int \widetilde{W}\left(z^{\prime}, k\right), \quad k \in \mathcal{C}_{a}
$$

see (3.16)-(3.23) for a precise definition of $J(z, k)$. The path of integration in the above integral is the subarc from $z_{r}$ to $k$ on $\mathcal{C}_{a}$. It lies in $\left\{\operatorname{Im} z \geq \operatorname{Im} z_{r}\right\}$ when $\operatorname{Im} k \geq \operatorname{Im} z_{r}$ and in $\left\{\operatorname{Im} z \leq \operatorname{Im} z_{r}\right\}$ when $\operatorname{Im} k \leq \operatorname{Im} z_{r}$. The definitions of $J^{0}$ and the differential form $\widetilde{W}$ involve the square root

$$
\lambda\left(z^{\prime}, k\right)=\sqrt{\left(k-z^{\prime}\right)\left(k+\bar{z}^{\prime}\right)},
$$

with a branch cut along the segment $\left(-\bar{z}^{\prime}, z^{\prime}\right)$. We choose the determination of $\lambda\left(z_{r}, k\right)$ that behaves like $k$ (resp. $-k$ ) at infinity when $\operatorname{Im} k \geq \operatorname{Im} z_{r}$ (resp. $\operatorname{Im} k \leq \operatorname{Im} z_{r}$ ) and then keep a continuous determination of $\lambda\left(z^{\prime}, k\right)$ when $z^{\prime}$ moves along the path of integration. 
Remark 1.4. Making use of the symmetry principles (1.7) and (1.8), one deduces easily from Theorems 1.1 and 1.3 similar integral representations for the solutions of (1.4), that is in the case of a positive integer coefficient $\alpha$.

The second result concerns the correspondance between the Dirichlet and Neumann data $u_{t}$ and $u_{n}$. Having a Lax pair is equivalent to the existence of a closed differential form. By Poincaré lemma, this leads to the vanishing of an integral on a closed contour, the so-called global relation. In the present case, it will follow from results in Section 2 that

$$
\int_{\mathcal{C}_{a}}[(k-z)(k+\bar{z})]^{\alpha / 2-1}\left((k-i y) u_{t}(z)+i x u_{n}(z)\right) d s=0, \quad k \in \mathbb{C} \backslash\left(\mathcal{D}_{a} \cup \mathcal{D}_{-a}\right) .
$$

It is sometimes conjectured that there always exists at least one global relation (i.e. a Lax pair) that allows for the recovering of one type of boundary values from the other one. In the case of an even coefficient $\alpha$, we show that, indeed, this correspondance can be performed explicitly from such a relation.

Theorem 1.5. Assume $\alpha=-2(m-1), m \in \mathbb{N}^{*}$, and $u_{t}$ is a given function in $L^{2}\left(\mathcal{C}_{a}\right)$. Let $u_{n}$ be a function in $L^{2}\left(\mathcal{C}_{a}\right)$ such that the global relation (1.14) holds true. Then, $u_{n}$ is unique and can be explicitly recovered from that relation. A similar statement holds when $\alpha=2(m+1), m \in \mathbb{N}$. In that case, (1.14) does not allow the reconstruction of $u_{n}$. A relation that works is obtained by integrating another differential form, see (2.6).

Remark 1.6. Making use of the equation conjugate to (1.6), that is changing $\alpha$ into $-\alpha$, we derive the converse reconstruction of the Dirichlet data $u_{t}$ from the Neumann data $u_{n}$, still when $\alpha \in 2 \mathbb{Z}$ is an even coefficient. In Theorem 1.5 , it is actually not necessary to assume smoothness of the functions $u_{t}$ and $u_{n}$ on the boundary circle $\mathcal{C}_{a}$. Hence, we only assume these functions to be $L^{2}$ on $\mathcal{C}_{a}$.

Apparently, the reconstruction of $u_{n}$ from (1.14), or a similar relation, is not completely straightforward. It may be conjectured that the reconstruction of $u_{n}$ should be possible by an integral transform, like one of the Abel type, see e.g. [25]. We were not able to find such an integral transform in the present case. Our method uses rather the symmetry involved in the problem and the property of a particular linear differential equation stemming from the computation of a contour integral by Cauchy formula.

In Section 2 we recall the notion of Lax pairs and compute such pairs for the equation (1.6). We also briefly discuss the use of a Lax pair, or the related global relation, in deriving an explicit correspondance between Dirichlet and Neumann data in the simple case of the Laplace equation. The study of two specific Riemann-Hilbert problems, leading to the proofs of Theorems 1.1 and 1.3, is performed in Sections 3. The proof of Theorem 1.5 is displayed in Section 4.

\section{Lax pairs and closed differential forms}

A Lax pair for a partial differential equation $P(u)=0$ is a pair of ordinary differential equations, for a function $\phi$ related to $u$, which are compatible precisely when $P(u)=0$. 
The general equation (1.6) admits such a Lax pair. Indeed, writing (1.6) with respect to the complex variables $z$ and $\bar{z}$, we get

$$
u_{z \bar{z}}+\frac{\alpha}{2(z+\bar{z})}\left(u_{z}+u_{\bar{z}}\right)=0
$$

and a possible way to find a Lax pair is by rewriting (2.1) in the form

$$
\left(f(z, \bar{z}) u_{\bar{z}}\right)_{z}+\left(g(z, \bar{z}) u_{z}\right)_{\bar{z}}=0
$$

where the functions $f$ and $g$ have to be determined. Expanding (2.2) and comparing with (2.1), we get

$$
f_{z}=\frac{\alpha}{2(z+\bar{z})}(f+g), \quad g_{\bar{z}}=\frac{\alpha}{2(z+\bar{z})}(f+g) .
$$

Differentiating the first equation with respect to $\bar{z}$, the second one with respect to $z$, and adding, we obtain

$$
(f+g)_{z \bar{z}}=\frac{\alpha}{2(z+\bar{z})}\left((f+g)_{z}+(f+g)_{\bar{z}}\right)-\frac{\alpha}{(z+\bar{z})^{2}}(f+g),
$$

so that $f+g$ satisfies the adjoint equation of (2.1) (see e.g. [9, Chapter 7] for the notion of the adjoint equation). We seek solutions in the form

$$
(f+g)(z, \bar{z})=(z+\bar{z}) A(z) B(\bar{z}) .
$$

Plugging that in the previous equation leads to

$$
(z+\bar{z}) A_{z} B_{\bar{z}}=(\alpha / 2-1)\left(A B_{\bar{z}}+A_{z} B\right)
$$

and thus

$$
-(\alpha / 2-1) A / A_{z}+z=k=(\alpha / 2-1) B / B_{\bar{z}}-\bar{z},
$$

where $k$ may be any complex number. It is a new, additional parameter in the problem, the so-called spectral parameter. Solving for the two equations, we get, as possible solutions,

$$
A(z)=(k-z)^{\alpha / 2-1}, \quad B(\bar{z})=-(k+\bar{z})^{\alpha / 2-1} .
$$

In view of (2.3), we may thus choose for $f$ and $g$,

$$
f(z, \bar{z})=(k-z)^{\alpha / 2}(k+\bar{z})^{\alpha / 2-1}, \quad g(z, \bar{z})=-(k-z)^{\alpha / 2-1}(k+\bar{z})^{\alpha / 2} .
$$

Hence, (2.1) is equivalent to

$$
\left((k+\bar{z})^{\alpha / 2-1}(k-z)^{\alpha / 2} u_{\bar{z}}\right)_{z}-\left((k+\bar{z})^{\alpha / 2}(k-z)^{\alpha / 2-1} u_{z}\right)_{\bar{z}}=0 .
$$

This last equation is equivalent to the compatibility of the two ordinary differential equations

$$
\phi_{z}(z, k)=(k+\bar{z})^{\alpha / 2}(k-z)^{\alpha / 2-1} u_{z}(z), \quad \phi_{\bar{z}}(z, k)=(k+\bar{z})^{\alpha / 2-1}(k-z)^{\alpha / 2} u_{\bar{z}}(z),
$$

which thus gives a Lax pair for (2.1). Equivalently, we may express the property of the Lax pair as the closedness of a differential form. 
Proposition 2.1. The function u satisfies (2.1) in a bounded, simply connected domain $\Omega$ of $\mathbb{H}$ if and only if the differential form

$$
z \mapsto W(z, k)=[(k-z)(k+\bar{z})]^{\alpha / 2-1}\left[(k+\bar{z}) u_{z}(z) d z+(k-z) u_{\bar{z}}(z) d \bar{z}\right]
$$

is closed in $\Omega$. Note that, when $\alpha \in 2 \mathbb{N}^{*}$, the differential form has no singularities in $\Omega$ and $k$ can be any complex number. Otherwise, for $\alpha \in \mathbb{R} \backslash 2 \mathbb{N}^{*}, W(z, k)$ has either a pole or a branch point at $k$ and $-\bar{k}$, so that $k$ should lie outside $\Omega$ and $-\bar{\Omega}$.

Making use of the symmetry relation (1.8), we derive a second Lax pair, namely

$$
\begin{aligned}
\phi_{z}(z, k) & =(k+\bar{z})^{1-\alpha / 2}(k-z)^{-\alpha / 2}\left((z+\bar{z})^{\alpha-1} u\right)_{z}(z) \\
& =(k+\bar{z})^{1-\alpha / 2}(k-z)^{-\alpha / 2}(z+\bar{z})^{\alpha-2}\left[(z+\bar{z}) u_{z}(z)+(\alpha-1) u(z)\right], \\
\phi_{\bar{z}}(z, k) & =(k+\bar{z})^{-\alpha / 2}(k-z)^{1-\alpha / 2}\left((z+\bar{z})^{\alpha-1} u\right)_{\bar{z}}(z) \\
& =(k+\bar{z})^{-\alpha / 2}(k-z)^{1-\alpha / 2}(z+\bar{z})^{\alpha-2}\left[(z+\bar{z}) u_{\bar{z}}(z)+(\alpha-1) u(z)\right],
\end{aligned}
$$

and the related closed form

$$
\begin{aligned}
& z \mapsto W(z, k)=[(k-z)(k+\bar{z})]^{-\alpha / 2} x^{\alpha-2} \\
& \cdot\left[(k+\bar{z})\left(2 x u_{z}(z)+(\alpha-1) u(z)\right) d z+(k-z)\left(2 x u_{\bar{z}}(z)+(\alpha-1) u(z)\right) d \bar{z}\right] .
\end{aligned}
$$

This second differential form will be useful in Section 4.

We end this section with a brief discussion on the possible use of a Lax pair for the derivation of an explicit correspondance between different types of boundary data. It is easy to check that not all Lax pairs can achieve this goal. For instance, if we consider (2.4) when $m=0$, we get

$$
\phi_{z}(z, k)=u_{z}(z) /(k-z), \quad \phi_{\bar{z}}(z, k)=u_{\bar{z}}(z) /(k+\bar{z}),
$$

which is a Lax pair for the Laplace equation $\Delta u=0$. If we choose as a domain $\Omega$ the unit disk $\mathbb{D}$, the differential form $W(z, k)$ from Proposition 2.1 is closed in $\mathbb{D}$ and thus we get, in terms of the tangent and normal derivatives $u_{t}$ and $u_{n}$ on the unit circle,

$$
\int_{\partial \mathbb{D}} \frac{x u_{n}(z)}{(k-z)(k+\bar{z})} d s=\int_{\partial \mathbb{D}} \frac{(y+i k) u_{t}(z)}{(k-z)(k+\bar{z})} d s, \quad k \in \mathbb{C} \backslash \mathbb{D} .
$$

Assume that the tangent derivative $u_{t}$ is known, hence also the integral in the right-hand side, equal to some function $\psi(k)$. Decomposing the real function $x u_{n}(z)=g(z)+\bar{g}(1 / z)$, with $g(z)$ analytic in $\mathbb{D}, g(0) \in \mathbb{R}$, it is readily checked that (2.7) rewrites as

$$
\bar{g}(1 / k)-g(-1 / k)=k \psi(k)
$$

which allows one to recover only the half of $g$, namely the imaginary parts of its even Taylor coefficients and the real parts of the odd ones.

On the contrary, when considering the domain $\mathcal{D}_{a}=D(a, 1)$ instead of $\mathbb{D}$, we will see in Section 4 that the Lax pair in (2.4) is sufficient to recover the Dirichlet to Neumann correspondance. 


\section{Characterization by a Riemann-Hilbert problem}

The goal of this section is to prove Theorems 1.1 and 1.3.

\subsection{Case of a negative even integer coefficient $\alpha=-2 m, m \in \mathbb{N}$}

Proof of Theorem 1.1. The differential form $W(z, k)$ rewrites as

$$
W(z, k)=((k-z)(k+\bar{z}))^{-m-1}\left((k+\bar{z}) u_{z}(z) d z+(k-z) u_{\bar{z}}(z) d \bar{z}\right) .
$$

Equations (2.4) can be written as $d \phi=W$ and we thus construct a function $\phi$ of the form

$$
\phi(z, k)=\int_{z_{r}}^{z} W\left(z^{\prime}, k\right)
$$

where the path of integration needs to be defined. For $k$ such that $\operatorname{Im} k \geq \operatorname{Im} z$, we integrate from $z_{r}$ to $z$ following the lower part $\mathcal{C}_{\text {low }}$ of the circle $\mathcal{C}_{a}$ and then the segment from $z_{l}$ to $z$. For $k$ such that $\operatorname{Im} k \leq \operatorname{Im} z$, we integrate from $z_{r}$ to $z$ following the upper part of the circle $\mathcal{C}_{u p}$ and then again the segment from $z_{l}$ to $z$, see Figure 1 . This defines $\phi(z, k)$ as an

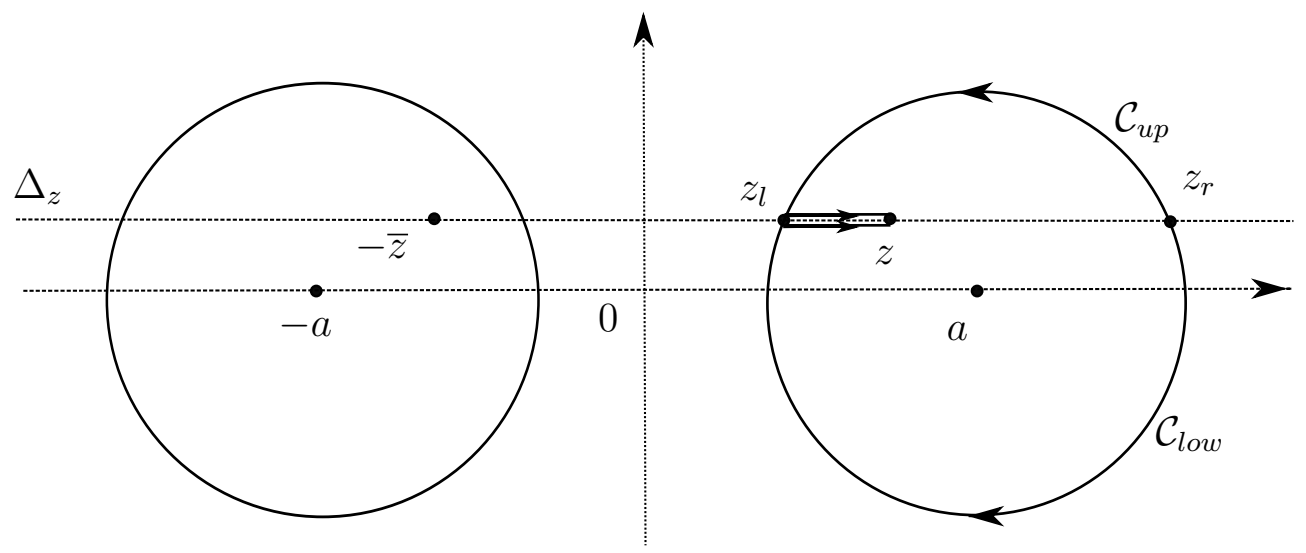

Figure 1: The paths of integration $\gamma_{1}$ and $\gamma_{2}$ from $z_{r}$ to $z$, respectively along $\mathcal{C}_{\text {up }}$ and $\mathcal{C}_{\text {low }}$ for the definition $(3.2)$ of $\phi(z, k)$.

analytic function of $k$ outside of the line $\Delta_{z}$ through $z$ and $-\bar{z}$ where it may possibly have a jump. We remark from (3.1) and (3.2) that the function $k \mapsto \phi(z, k)$ has poles of order $m$ at $k \in\left\{-\bar{z}_{r},-\bar{z}, z, z_{r}\right\}$ if $m>0$ and logarithmic singularities if $m=0$. It also satisfies the symmetry relation

$$
\phi(z,-\bar{k})=-\overline{\phi(z, k)} .
$$

In the sequel, we use the notations $\phi^{+}(z, k)$ and $\phi^{-}(z, k)$ for the limit values of $\phi(z, k)$ when $k$ tends to the left and right of a given arc, according to its orientation. The fact that the differential form $W\left(z^{\prime}, k\right)$ is closed in the disk $\mathcal{D}_{a}$ when $k$ lies outside of that disk implies that there is no jump

$$
J(z, k):=\phi^{+}(z, k)-\phi^{-}(z, k)
$$


of $\phi(z, k)$ on the part of $\Delta_{z}$ outside of $\mathcal{D}_{a}$ and $-\mathcal{D}_{a}$. For $k$ inside the disks, we have

$$
\begin{array}{ll}
J(z, k)=-\int_{\mathcal{C}_{a}} W\left(z^{\prime}, k\right), & k \in\left(z, z_{r}\right) \cup\left(-\bar{z}_{r},-\bar{z}\right), \\
J(z, k)=0, & k \in\left(z_{l}, z\right) \cup\left(-\bar{z},-\bar{z}_{l}\right) .
\end{array}
$$

For computing the jump on $\left(-\bar{z}_{r},-\bar{z}\right)$, we have used the symmetry (3.3). For the jump on $\left(z_{l}, z\right)$, we have deformed the two paths of integration into the segment $\left(z_{r}, z\right)$ which is possible by closedness of the differential form $W\left(z^{\prime}, k\right)$. Note that $k \mapsto J(z, k)$ has no singularity in $z$ and $z_{r}$. In $z$ it is clear, while in $z_{r}$, it can be seen by performing $m$ integrations by parts of $W\left(z^{\prime}, k\right)$ on the closed contour $\mathcal{C}_{a}$, see also [10, Section 4.4] for a similar computation. Concerning the function $k \mapsto \phi(z, k)$, as said before, it has poles of order $m$ at $\left\{z, z_{r},-\bar{z},-\bar{z}_{r}\right\}$ if $m>0$ and logarithmic singularities if $m=0$. Moreover, at infinity, it behaves like

$$
\phi(z, k) \rightarrow k^{-2 m-1}\left(u(z)-u\left(z_{r}\right)\right), \quad \text { as } k \rightarrow \infty .
$$

If $m>0$, we renormalize the problem at infinity by defining

$$
\begin{aligned}
& \widetilde{\phi}(z, k)=((k-z)(k+\bar{z}))^{m} \phi(z, k), \\
& \widetilde{J}(z, k)=((k-z)(k+\bar{z}))^{m} J(z, k) .
\end{aligned}
$$

Then, the function $k \mapsto \widetilde{\phi}(z, k)$ behaves like $k^{-1}\left(u(z)-u\left(z_{r}\right)\right)$ at infinity and is regular at $z$ and $-\bar{z}$, except for logarithmic singularities when $m=0$. It still has polar singularities at $z_{r}$ and $-\bar{z}_{r}$. Let us denote by $\widetilde{\phi}_{z_{r},-\bar{z}_{r}}(z, k)$ its polar part at these points, that is the sum of the terms of negative degrees in the Laurent expansions of $\widetilde{\phi}(z, k)$ at $z_{r}$ and $-\bar{z}_{r}$. The function $\widetilde{\phi}-\widetilde{\phi}_{z_{r},-\bar{z}_{r}}$ is analytic outside of the segments $\left(z, z_{r}\right)$ and $\left(-\bar{z}_{r},-\bar{z}\right)$ where it has the jump $\widetilde{J}(z, k)$. It has at most logarithmic singularities at $\left\{z, z_{r},-\bar{z},-\bar{z}_{r}\right\}$ and vanishes at infinity.

These properties completely determine the function $\widetilde{\phi}-\widetilde{\phi}_{z_{r},-\bar{z}_{r}}$. Indeed, if there were another function with these properties, their difference would be entire and vanishing at infinity, hence the zero function. Thanks to the Plemelj formula, we have an integral expression for $\widetilde{\phi}(z, k)-\widetilde{\phi}_{z_{r},-\bar{z}_{r}}(z, k)$, namely,

$$
\widetilde{\phi}(z, k)-\widetilde{\phi}_{z_{r},-\bar{z}_{r}}(z, k)=\frac{1}{2 i \pi} \int_{\left(-\bar{z}_{r},-\bar{z}\right) \cup\left(z, z_{r}\right)} \frac{\widetilde{J}\left(z, k^{\prime}\right)}{k^{\prime}-k} d k^{\prime} .
$$

Denoting by $a_{r}$ and $a_{-r}$ the residues of $\widetilde{\phi}(z, k)$ at $k=z_{r}$ and $k=-\bar{z}_{r}$ respectively, and equating the coefficients of $k^{-1}$ in the expansion of (3.6) at infinity, we get

$$
\begin{aligned}
u(z)-u\left(z_{r}\right) & =a_{r}+a_{-r}-\frac{1}{2 i \pi} \int_{\left(-\bar{z}_{r},-\bar{z}\right) \cup\left(z, z_{r}\right)} \widetilde{J}\left(z, k^{\prime}\right) d k^{\prime} \\
& =2 \operatorname{Re}\left(a_{r}\right)-\frac{1}{\pi} \operatorname{Im} \int_{\left(z, z_{r}\right)} \widetilde{J}\left(z, k^{\prime}\right) d k^{\prime},
\end{aligned}
$$


where we have used the symmetry relation $\widetilde{\phi}(z,-\bar{k})=-\overline{\widetilde{\phi}(z, k)}$. It remains to show that the residue $a_{r}$ can be explicitly computed from the knowledge of the derivatives $u_{t}$ and $u_{n}$ on $\mathcal{C}_{a}$. From (3.4), it is sufficient to know the polar part of $\phi(z, k)$ at $z_{r}$. To compute this polar part, we first rewrites $W(z, k)$, defined in (3.1), by expressing the complex derivatives in terms of the tangential and (outer) normal derivatives on the circle $\mathcal{C}_{a}$,

$$
u_{z} d z=\frac{1}{2}\left(u_{t}+i u_{n}\right) d s, \quad u_{\bar{z}} d \bar{z}=\frac{1}{2}\left(u_{t}-i u_{n}\right) d s,
$$

with $d s$ the length element on $\mathcal{C}_{a}$. We get, with $z=x+i y \in \mathcal{C}_{a}$,

$$
\begin{aligned}
W(z, k) & =((k-z)(k+\bar{z}))^{-m-1}\left((k-i y) u_{t}(z)+i x u_{n}(z)\right) d s, \\
& =(k-z)^{-m-1} w(z, k) d z
\end{aligned}
$$

where

$$
w(z, k):=(k+\bar{z})^{-m-1}\left((k-i y) u_{t}(z)+i x u_{n}(z)\right) \tau^{-1}(z)
$$

and $\tau(z)$ denotes the unit vector tangent to $\mathcal{C}_{a}$ at the point $z$. Let us set

$$
\widetilde{\partial}_{t} f:=\tau^{-1}(z) \partial_{t} f
$$

for a function $f$ on $\mathcal{C}_{a}$. Because of analiticity,

$$
\partial_{z}(k-z)^{-j-1} d z=\partial_{t}(k-z)^{-j-1} d s=\widetilde{\partial}_{t}(k-z)^{-j-1} d z, \quad j=m-1, \ldots, 0 .
$$

Hence, performing $m$ integrations by parts on the integral in (3.2), we obtain

$$
\begin{aligned}
\phi(z, k)= & c_{0}\left[\left(k-z^{\prime}\right)^{-m} w\left(z^{\prime}, k\right)\right]_{z_{r}}^{z}+\cdots \\
& +c_{m-1}\left[\left(k-z^{\prime}\right)^{-1} \widetilde{\partial}_{t}^{(m-1)} w\left(z^{\prime}, k\right)\right]_{z_{r}}^{z}-c_{m-1} \int_{z_{r}}^{z}\left(k-z^{\prime}\right)^{-1} \widetilde{\partial}_{t}^{(m)} w\left(z^{\prime}, k\right) d z^{\prime}
\end{aligned}
$$

where

$$
c_{j}=(-1)^{j} \Gamma(m-j) / \Gamma(m+1), \quad j=0, \ldots, m-1 .
$$

The polar part of $\phi(z, k)$ at $z_{r}$ can be read in the bracketed terms in (3.9) as

$$
-c_{0} \sum_{j=0}^{m-1} \frac{\partial_{k}^{(j)} w\left(z_{r}, z_{r}\right)}{j !\left(k-z_{r}\right)^{m-j}}-\cdots-c_{m-1} \frac{\widetilde{\partial}_{t}^{(m-1)} w\left(z_{r}, z_{r}\right)}{k-z_{r}}
$$

where $\partial_{k}$ denotes the operator of differentiation with respect to the variable $k$. This finishes the proof of Theorem 1.1.

\subsection{Case of a negative odd integer coefficient $\alpha=-2 m+1, m \in \mathbb{N}$}

Proof of Theorem 1.3. The differential form (2.5) now rewrites as

$$
W(z, k)=((k-z)(k+\bar{z}))^{-m-1 / 2}\left((k+\bar{z}) u_{z}(z) d z+(k-z) u_{\bar{z}}(z) d \bar{z}\right) .
$$


The novelty with respect to the even coefficient case is that $W(z, k)$ involves the square root

$$
\lambda(z, k)=\sqrt{(k-z)(k+\bar{z})}
$$

which, as a function of $k$, is defined on a Riemann surface $\mathcal{S}_{z}$ of genus 0 , consisting of two copies of $\mathbb{C}$, denoted by $\mathcal{S}_{z, 1}$ for the upper sheet, and by $\mathcal{S}_{z, 2}$ for the lower sheet. The two sheets are glued together along a branch cut from $z$ to $-\bar{z}$, that we choose to be the horizontal segment $(-\bar{z}, z)$. We denote by $\lambda_{1}$ and $\lambda_{2}$ the determination of the square root $\lambda$, as a function of $k$, on the upper and lower sheets of $\mathcal{S}_{z}$ where we assume that

$$
\begin{aligned}
& \lambda_{1}(z, k)=k(1+\mathcal{O}(1 / k)), \quad \text { as } k \rightarrow \infty_{1} \text { on the upper sheet } \mathcal{S}_{z, 1}, \\
& \lambda_{2}(z, k)=-k(1+\mathcal{O}(1 / k)), \quad \text { as } k \rightarrow \infty_{2} \text { on the lower sheet } \mathcal{S}_{z, 2} .
\end{aligned}
$$

We also denote by $W_{1}$ and $W_{2}$ the values of the differential form $W$ corresponding to the determinations $\lambda_{1}$ and $\lambda_{2}$ of the square root. For future use, we remark that the function $\lambda(z, k)$ and the differential form $W(z, k)$ satisfy the following symmetry relations:

$$
\lambda(z,-\bar{k})=-\overline{\lambda(z, k)}, \quad W(z,-\bar{k})=\overline{W(z, k)} .
$$

As in the previous section, we construct a function $\phi$ of the form

$$
\phi(z, k)=\int_{z_{r}}^{z} W\left(z^{\prime}, k\right),
$$

where the path of integration needs to be defined. Similarly to the method applied in [20], we define, for each $z$, the function $k \rightarrow \phi(z, k)$ as a map from the Riemann surface $\mathcal{S}_{z}$ to $\mathbb{C}$. The path of integration in (3.14) is chosen to be $\gamma_{1}$ when $k \in \mathcal{S}_{z, 1}$ and $\gamma_{2}$ when $k \in \mathcal{S}_{z, 2}$, see Figures 2 and 3 . Note that, when $k$ lies outside of the convex hull of the two

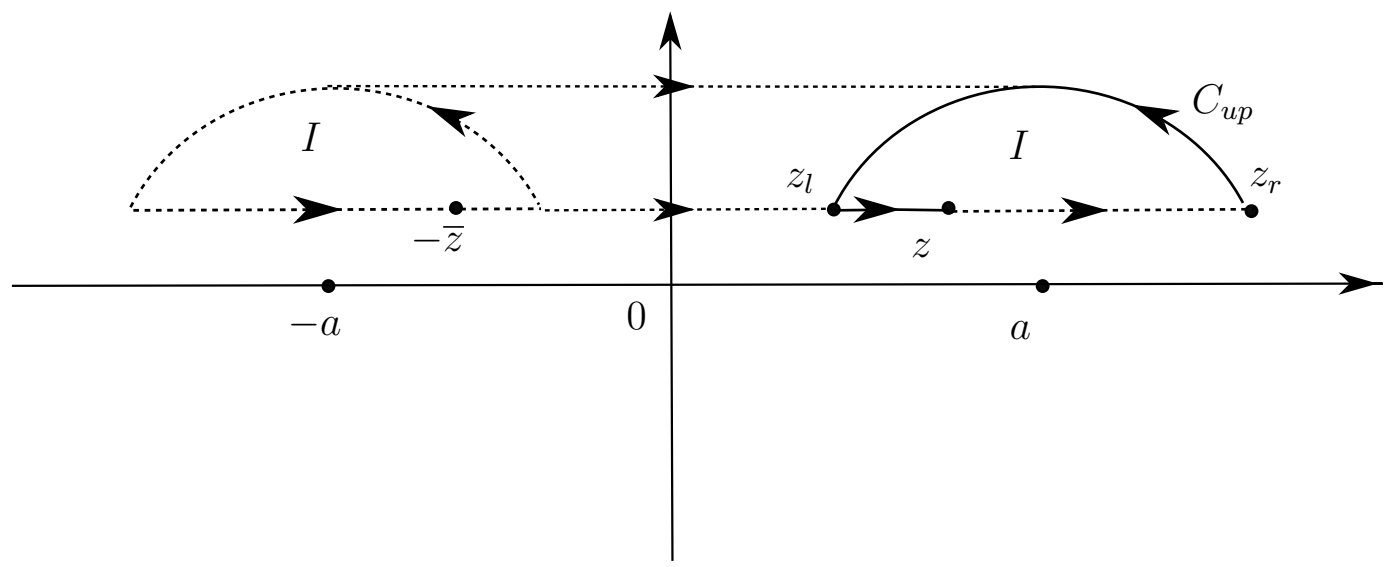

Figure 2: Orientation of contours and path of integration $\gamma_{1}$ (solid line) for the definition (3.14) of $\phi(z, k)$ on the upper sheet $\mathcal{S}_{z, 1}$. The path starts at $z_{r}$, goes along the upper part $\mathcal{C}_{u p}$ of the circle $\mathcal{C}_{a}$ to $z_{l}$ and then follows the horizontal segment up to $z$. The choice of the determination of the square root $\lambda$ at the initial point $z_{r}$ of the path $\gamma_{1}$ depends on the region which contains $k$. 


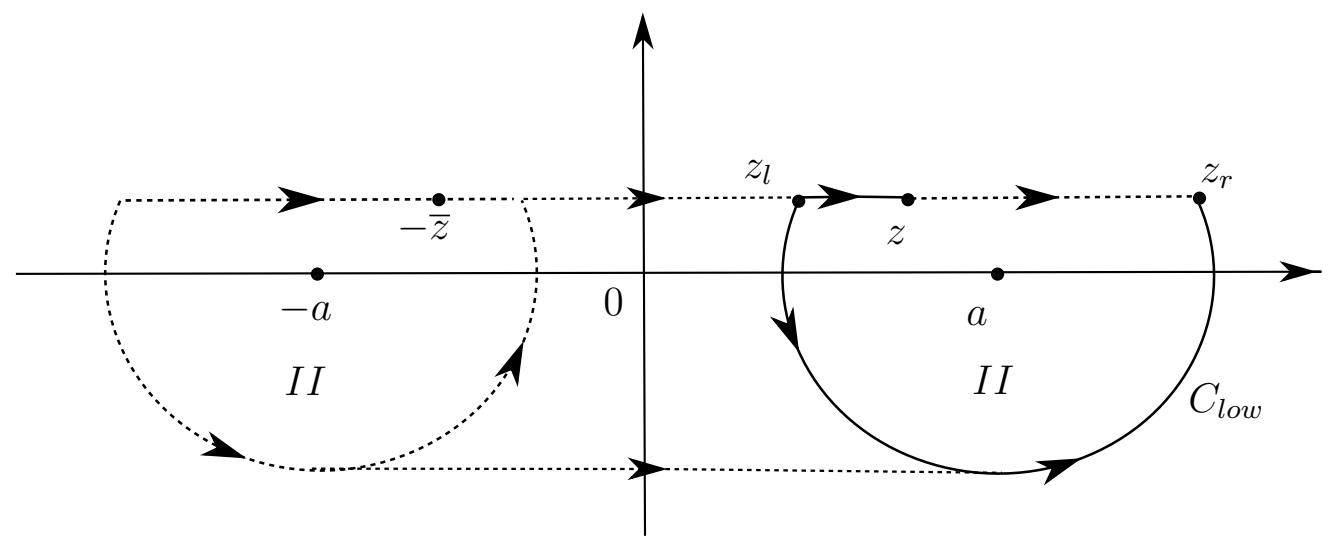

Figure 3: Orientation of contours and path of integration $\gamma_{2}$ (solid line) for the definition (3.14) of $\phi(z, k)$ on the lower sheet $\mathcal{S}_{z, 2}$. The path starts at $z_{r}$, goes along the lower part $\mathcal{C}_{\text {low }}$ of the circle $\mathcal{C}_{a}$ to $z_{l}$ (thus describes $\mathcal{C}_{\text {low }}$ clockwise) and then follows the horizontal segment up to $z$. The choice of the determination of the square root $\lambda$ at the initial point $z_{r}$ of the path $\gamma_{2}$ depends on the region which contains $k$.

symmetric circles, the branch cut from $z^{\prime}$ to $-\bar{z}^{\prime}$ never intersects $k$ as $z^{\prime}$ goes from $z_{r}$ to $z$ along $\gamma_{1}$ or $\gamma_{2}$. For such $k$, we may therefore use the same determination of the square root $\lambda\left(z^{\prime}, k\right)$ for computing the integral (3.14). When $k$ lies inside one of the circles (i.e. regions I and II in Figures 2 and 3), the branch cut intersects $k$ once. Hence, for $k \in \mathcal{S}_{z, 1}$ lying in the upper part of one of the two circles, we use the determination of the square root $\lambda_{2}\left(z^{\prime}, k\right)$ on the first part of integration, before the crossing, and the determination $\lambda_{1}\left(z^{\prime}, k\right)$ after the crossing, and conversely for $k \in \mathcal{S}_{z, 2}$ lying in the lower part of one of the two circles. Finally, when $k$ lies between the two circles, the branch cut intersects $k$ twice, so that, in this case, we start integration with the determination of the square root $\lambda\left(z^{\prime}, k\right)$ corresponding to $k$, then change to the other determination after the first crossing, and come back to the first determination after the second crossing.

The function $\phi(z, k)$ is an analytic function of $k \in \mathcal{S}_{z}$ outside of arcs where it has jumps. Note that, in view of (3.10), it has poles of order $m$ at $k \in\left\{z, z_{r},-\bar{z},-\bar{z}_{r}\right\}$.

As in the previous section, $\phi^{+}(z, k)$ and $\phi^{-}(z, k)$ denote the limit values of $\phi(z, k)$ when $k$ tends to the left and right of a given contour, according to its orientation. Let us compute the jumps,

$$
J(z, k)=\phi^{+}(z, k)-\phi^{-}(z, k),
$$

of the function $\phi(z, k)$ on the different arcs shown in Figures 2 and 3. As in the previous section, we denote by $\mathcal{C}_{u p}$ the part of the circle $\mathcal{C}_{a}=C(a, 1)$ above the segment $\left(z_{l}, z_{r}\right)$ and by $\mathcal{C}_{\text {low }}$ the part of the circle $\mathcal{C}_{a}$ below that segment. For $k$ on one of the two circles, we denote by $\widetilde{k}$ the other point on the circle with the same imaginary part, that is, we have

$$
\begin{array}{ll}
\widetilde{k}-a=-\overline{(k-a)}, & k \in \mathcal{C}_{a} \\
\widetilde{k}+a=-\overline{(k+a)}, & k \in-\mathcal{C}_{a} .
\end{array}
$$

To compute the jumps $J(z, k)$ we first rewrites $W(z, k)$, defined in $(3.10)$, in terms of $d s$, the length element on $\mathcal{C}_{a}$. By the same computation as the one in the previous section, we 
now get, with $z=x+i y \in \mathcal{C}_{a}$,

$$
\begin{aligned}
W(z, k) & =((k-z)(k+\bar{z}))^{-m-1 / 2}\left((k-i y) u_{t}(z)+i x u_{n}(z)\right) d s \\
& =(k-z)^{-m-1 / 2} w(z, k) d z
\end{aligned}
$$

where

$$
w(z, k):=(k+\bar{z})^{-m-1 / 2}\left((k-i y) u_{t}(z)+i x u_{n}(z)\right) \tau^{-1}(z)
$$

and $\tau(z)$ still denotes the unit vector tangent to $\mathcal{C}_{a}$ at the point $z$. Performing $m$ integrations by parts on the integral in (3.14), we obtain in a similar way as in the previous section,

$$
\begin{aligned}
\phi(z, k) & =c_{0}\left[\left(k-z^{\prime}\right)^{-m+1 / 2} w\left(z^{\prime}, k\right)\right]_{z_{r}}^{z}+\cdots \\
+ & c_{m-1}\left[\left(k-z^{\prime}\right)^{-1 / 2} \widetilde{\partial}_{t}^{(m-1)} w\left(z^{\prime}, k\right)\right]_{z_{r}}^{z}-c_{m-1} \int_{z_{r}}^{z}\left(k-z^{\prime}\right)^{-1 / 2} \widetilde{\partial}_{t}^{(m)} w\left(z^{\prime}, k\right) d z^{\prime},
\end{aligned}
$$

where

$$
c_{j}=(-1)^{j} \Gamma(m-1 / 2-j) / \Gamma(m+1 / 2), \quad j=0, \ldots, m-1,
$$

and the operator $\widetilde{\partial}_{t} f$ is still defined by (3.8). The bracketed terms contain the polar parts of $\phi(z, k)$, of degree $m$, at $z$ and $z_{r}$. Note that the last integral converges when $k \in \mathcal{C}_{a}$. Assume $k \in \mathcal{S}_{z, 1}$ and lies on the right half of $\mathcal{C}_{u p}$. Then, from the above definition of $\phi$, we derive that

$$
J(z, k)=J_{1}^{0}\left(z_{r}, k\right)+\int_{z_{r}}^{k} \widetilde{W}_{1}\left(z^{\prime}, k\right),
$$

with

$$
\begin{gathered}
J_{1}^{0}\left(z_{r}, k\right)=2 \sum_{j=0}^{m-1} c_{j}\left(k-z_{r}\right)^{j-m+1 / 2} \widetilde{\partial}_{t}^{(j)} w_{1}\left(z_{r}, k\right), \\
\widetilde{W}_{1}\left(z^{\prime}, k\right)=2 c_{m-1}\left(k-z^{\prime}\right)^{-1 / 2} \widetilde{\partial}_{t}^{(m)} w_{1}\left(z^{\prime}, k\right) d z^{\prime}
\end{gathered}
$$

where the subscript 1 in the above expressions means that we use the determination $\lambda_{1}$ of the square root to evaluate them. The jumps on the left half of $\mathcal{C}_{\text {up }}$ on $\mathcal{S}_{z, 1}$ and on $\mathcal{C}_{\text {low }}$ on $\mathcal{S}_{z, 2}$ can be computed in the same way. The jumps on $-\overline{\mathcal{C}}_{\text {up }}$ and $-\overline{\mathcal{C}}_{\text {low }}$ can be derived from the symmetry relation satisfied by $\phi(z, k)$,

$$
\phi(z,-\bar{k})=\overline{\phi(z, k)}
$$

The result is as follows. For $k \in \mathcal{S}_{z, 1}$,

$$
\begin{array}{ll}
J(z, k)=J_{1}^{0}\left(z_{r}, k\right)+\int_{\left(z_{r}, k\right)} \widetilde{W}_{1}\left(z^{\prime}, k\right), & k \text { on the right half of } \mathcal{C}_{u p}, \\
J(z, k)=J_{1}^{0}\left(z_{r}, k\right)+\int_{\left(z_{r}, \widetilde{k}\right)} \widetilde{W}_{1}\left(z^{\prime}, k\right)-\int_{(\widetilde{k}, k)} \widetilde{W}_{1}\left(z^{\prime}, k\right), & k \text { on the left half of } \mathcal{C}_{u p}, \\
J(z, k)=\overline{J(z,-\bar{k})}, & k \text { on }-\overline{\mathcal{C}}_{u p},
\end{array}
$$


where the path of integration $(a, b)$ in each of the above integrals is the subarc of $\mathcal{C}_{\text {up }}$ from $a$ to $b$, in the positive direction. For $k \in \mathcal{S}_{z, 2}$, we have

$$
\begin{array}{ll}
J(z, k)=J_{2}^{0}\left(z_{r}, k\right)+\int_{\left(z_{r}, k\right)} \widetilde{W}_{2}\left(z^{\prime}, k\right), & k \text { on the right half of } \mathcal{C}_{\text {low }}, \\
J(z, k)=J_{2}^{0}\left(z_{r}, k\right)+\int_{\left(z_{r}, \widetilde{k}\right)} \widetilde{W}_{2}\left(z^{\prime}, k\right)-\int_{(\widetilde{k}, k)} \widetilde{W}_{2}\left(z^{\prime}, k\right), & k \text { on the left half of } \mathcal{C}_{\text {low }}, \\
J(z, k)=\overline{J(z,-\bar{k}),} & k \text { on }-\overline{\mathcal{C}}_{\text {low }},
\end{array}
$$

where $(a, b)$ in each of the integrals denotes the subarc of $\mathcal{C}_{\text {low }}$ from $a$ to $b$, in the negative direction.

Note that the above jumps take place on two open contours on the Riemann surface $\mathcal{S}_{z}$. The first one starts at $z_{r, 1}$ on the first sheet, follows the circle $\mathcal{C}_{a}$, goes through the cut at $z_{l}$ and finishes at $z_{r, 2}$ on the second sheet. The second one start at $-\bar{z}_{r, 1}$ on the first sheet, follows the circle $-\mathcal{C}_{a}$, goes through the cut at $-\bar{z}_{l}$ and finishes at $-\bar{z}_{r, 2}$ on the second sheet. Note also that the jump on the first contour is continuous at $z_{l}$ (compare (3.19) and (3.22) at $\left.k=z_{l}\right)$, and, by symmetry, the same is true of the jump on the second contour at $-\bar{z}_{l}$.

It follows, simply from the definition $(3.14)$ of $\phi(z, k)$ and the choice of the determinations, that, on each sheet, $\phi(z, k)$ has no jumps on $\left(z, z_{r}\right)$ and $\left(-\bar{z}_{r},-\bar{z}\right)$. On the branch cut $(-\bar{z}, z)$ of the Riemann surface $\mathcal{S}_{z}$ there is also no jumps. Indeed, on the part $\left(-\bar{z}_{l}, z_{l}\right)$, $k$ is not close from the paths of integration and we can use the definition (3.14) to compute the jumps. One has

$$
\begin{aligned}
& \phi_{1}^{+}(z, k)-\phi_{2}^{-}(z, k)=\int_{z_{r}}^{z} W_{1}^{+}\left(z^{\prime}, k\right)-\int_{z_{r}}^{z} W_{2}^{-}\left(z^{\prime}, k\right)=0, \\
& \phi_{1}^{-}(z, k)-\phi_{2}^{+}(z, k)=\int_{z_{r}}^{z} W_{1}^{-}\left(z^{\prime}, k\right)-\int_{z_{r}}^{z} W_{2}^{+}\left(z^{\prime}, k\right)=0,
\end{aligned}
$$

where we have deformed the original paths of integration $\gamma_{1}$ and $\gamma_{2}$ into the segment $\left(z_{r}, z\right)$, which is possible since the differential form $W(z, k)$ is closed. When $k \in \mathcal{S}_{z, 1}$ is on the side of $\left(z_{l}, z\right)$ or $k \in \mathcal{S}_{z, 2}$ is on the + side of $\left(z_{l}, z\right)$, we may deform both paths $\gamma_{1}$ and $\gamma_{2}$ into the segment $\left(z_{r}, z\right)$ and we get

$$
\phi_{1}^{-}(z, k)-\phi_{2}^{+}(z, k)=\int_{z_{r}}^{z} W_{1}^{-}\left(z^{\prime}, k\right)-\int_{z_{r}}^{z} W_{2}^{+}\left(z^{\prime}, k\right)=0 .
$$

Finally, when $k \in \mathcal{S}_{z, 1}$ is on the + side of $\left(z_{l}, z\right)$ or $k \in \mathcal{S}_{z, 2}$ is on the - side of $\left(z_{l}, z\right)$, we deform both paths $\gamma_{1}$ and $\gamma_{2}$ into the segment $\left(z_{r}, k\right)$ followed by the segment $(k, z)$. Here $k$ is closed from the paths of integration, so we use (3.15) to compute the jumps. We get

$$
\begin{aligned}
\phi_{1}^{+}(z, k) & =-\frac{1}{2} \int_{z_{r}}^{k} \widetilde{W_{1}^{-}}\left(z^{\prime}, k\right)-\frac{1}{2} \int_{k}^{z} \widetilde{W_{1}^{+}}\left(z^{\prime}, k\right) \\
& =-\frac{1}{2} \int_{z_{r}}^{k} \widetilde{W_{2}^{+}}\left(z^{\prime}, k\right)-\frac{1}{2} \int_{k}^{z} \widetilde{W_{2}^{-}}\left(z^{\prime}, k\right)=\phi_{2}^{-}(z, k),
\end{aligned}
$$


where we note that the bracketed terms in (3.15) do not contribute to the jumps.

For completeness, let us remark that, if we would consider $\phi(z, k)$ as a function on one of the two sheets only, e.g. $\mathcal{S}_{z, 1}$, then it would have a jump on the segment $\left(-\bar{z}_{l}, z_{l}\right)$, considered as an arc on $\mathcal{S}_{z, 1}$, namely

$$
\int_{\mathcal{C}_{u p}} \widetilde{W}_{1}\left(z^{\prime}, k\right), \quad k \in\left(-\bar{z}_{l}, z_{l}\right) .
$$

On the second sheet $\mathcal{S}_{z, 2}$, a jump would also occurs,

$$
-\int_{\mathcal{C}_{\text {low }}} \widetilde{W}_{2}\left(z^{\prime}, k\right), \quad k \in\left(-\bar{z}_{l}, z_{l}\right),
$$

which is actually opposite to the previous one.

The term $J^{0}\left(z_{r}, k\right)$ has a polar singularity of order $m$ at $k=z_{r}$ so that the jumps (3.18)-(3.23) have polar singularities of order $m$ at either $z_{r}$ or $-\bar{z}_{r}$. Hence, instead of $\phi(z, k)$, we consider

$$
\widetilde{\phi}(z, k)=\left(\left(k-z_{r}\right)\left(k+\bar{z}_{r}\right)\right)^{m} \phi(z, k),
$$

whose jumps

$$
\widetilde{J}(z, k)=\left(\left(k-z_{r}\right)\left(k+\bar{z}_{r}\right)\right)^{m} J(z, k)
$$

are regular (and lie on the same contours as those of $\phi(z, k)$ ). From the definitions of $W$ and $\phi$, and in view of (3.11)-(3.12), we have

$$
\begin{aligned}
& \lim _{k \rightarrow \infty_{1}} \widetilde{\phi}(z, k)=\int_{z_{r}}^{z} d u=u(z)-u\left(z_{r}\right), \\
& \lim _{k \rightarrow \infty_{2}} \widetilde{\phi}(z, k)=-\int_{z_{r}}^{z} d u=u\left(z_{r}\right)-u(z),
\end{aligned}
$$

so that, in particular,

$$
\widetilde{\phi}\left(z, \infty_{1}\right)=-\widetilde{\phi}\left(z, \infty_{2}\right) .
$$

Next, the function $k \rightarrow \widetilde{\phi}(z, k)$ remains bounded near the four endpoints $z_{r, 1}, z_{r, 2},-\bar{z}_{r, 1}$, $-\bar{z}_{r, 2}$ of the two contours where the jumps (3.18)-(3.23) take place. Indeed, near $z_{r, 1}$, $W(z, k)$ is of order $\left(k-z_{r, 1}\right)^{-m-1 / 2}$ and consequently $\widetilde{\phi}(z, k)$ is of order $\left(k-z_{r, 1}\right)^{1 / 2}$. The same fact holds true near the three other points. As a last remark, let us mention that $\underset{\phi}{\phi}(z, k)$ has poles of order $m$ at $z$ and $-\bar{z}$ since this holds true for $\phi(z, k)$. We denote by $\widetilde{\phi}_{z,-\bar{z}}(z, k)$ the sum of its polar parts at $z$ and $-\bar{z}$.

The jumps (3.24), the relation (3.27) between the values at infinities and the boundedness near the endpoints completely characterize the function $\widetilde{\phi}-\widetilde{\phi}_{z,-\bar{z}}$ on $\mathcal{S}_{z}$. Indeed, if there are two such functions, then their difference would be analytic on the compact Riemann surface $\overline{\mathcal{S}}_{z}$, hence constant. Since it would also satisfy the relation (3.27), it could only be the zero function. An explicit expression can be given for the unique solution of the Riemann-Hilbert problem defined by the previous conditions (jumps, relation between the values at $\infty_{1}$ and $\infty_{2}$, and boundedness near the endpoints), namely 


$$
\begin{aligned}
\widetilde{\phi}(z, k)-\widetilde{\phi}_{z,-\bar{z}}(z, k)=\frac{1}{4 i \pi} \int_{\mathcal{C}_{u p} \cup-\overline{\mathcal{C}}_{u p}} \widetilde{J}\left(z, k^{\prime}\right)\left(\frac{\lambda(z, k)}{\lambda_{1}\left(z, k^{\prime}\right)}+1\right) \frac{d k^{\prime}}{k^{\prime}-k} \\
+\frac{1}{4 i \pi} \int_{\mathcal{C}_{\text {low } \cup-\overline{\mathcal{C}}_{\text {low }}}} \widetilde{J}\left(z, k^{\prime}\right)\left(\frac{\lambda(z, k)}{\lambda_{2}\left(z, k^{\prime}\right)}+1\right) \frac{d k^{\prime}}{k^{\prime}-k},
\end{aligned}
$$

where the contours of integration are oriented as in Figures 2 and 3. In the first integral $k^{\prime} \in \mathcal{S}_{z, 1}$ and in the second integral $k^{\prime} \in \mathcal{S}_{z, 2}$. Let us check that the expression in (3.28) satisfies the characterizing properties of $\widetilde{\phi}-\widetilde{\phi}_{z,-\bar{z}}$. Indeed, it defines an analytic function of $k$ on $\mathcal{S}_{z}$ outside of the two contours $\mathcal{C}_{\text {up }} \cup \mathcal{C}_{\text {low }}$ and $-\overline{\mathcal{C}}_{\text {up }} \cup-\overline{\mathcal{C}}_{\text {low }}$. Thanks to the Plemelj formula, we see that it has the right jumps on these contours. Because of (3.11)-(3.12), it also satisfies relation (3.27). From the formulas (3.18), (3.20), (3.21), (3.23), we get that $\widetilde{J}\left(z, k^{\prime}\right)$ vanishes at the endpoints of the two contours. Hence, the expression in (3.28) remains bounded near these endpoints, see [1, Lemma 7.2.2] for details.

Making use of (3.25), we deduce from (3.28), where we rename $k^{\prime}$ as $k$, that

$$
\begin{aligned}
u(z)-u\left(z_{r}\right) & =-\frac{1}{4 i \pi} \int_{\mathcal{C}_{\text {up }} \cup-\overline{\mathcal{C}}_{\text {up }}} \frac{\widetilde{J}(z, k)}{\lambda_{1}(z, k)} d k-\frac{1}{4 i \pi} \int_{\mathcal{C}_{\text {low } \cup-\overline{\mathcal{C}}_{\text {low }}}} \frac{\widetilde{J}(z, k)}{\lambda_{2}(z, k)} d k \\
& =-\frac{1}{2 \pi} \operatorname{Im} \int_{\mathcal{C}_{\text {up }}} \frac{\widetilde{J}(z, k)}{\lambda_{1}(z, k)} d k-\frac{1}{2 \pi} \operatorname{Im} \int_{\mathcal{C}_{\text {low }}} \frac{\widetilde{J}(z, k)}{\lambda_{2}(z, k)} d k \\
& =-\frac{1}{2 \pi} \operatorname{Im} \int_{\mathcal{C}_{a}} \frac{\widetilde{J}(z, k)}{\sqrt{(k-z)(k+\bar{z})}} d k .
\end{aligned}
$$

Note that the polar part $\widetilde{\phi}_{z,-\bar{z}}$ does not give any contribution in the above computation as $k$ tends to infinity. In the second equality we have used the first identity in (3.13) and the fact that

$$
\widetilde{J}(z,-\bar{k})=\overline{\widetilde{J}(z, k)}
$$

In the last expression, we integrate along the circle $\mathcal{C}_{a}$ oriented counter-clockwise, starting at $z_{r}$ with the determination of the square root that behaves like $k$ at infinity. At $k=z_{l}$, the determination changes so that the square root remains continuous along the path of integration. This finishes the proof of Theorem 1.3.

The method used in the proofs of Theorems 1.1 and 1.3 could be applied in case of a general, bounded, simply-connected domain $\Omega$ in the right half-plane $\mathbb{H}$. For the definition of the corresponding function $\phi(z, k)$, in particular the paths of integration $\gamma_{1}$ and $\gamma_{2}$, one could replace the points $z_{r}$ (resp. $z_{l}$ ) by e.g. a point $\omega_{r}$ (resp. $\omega_{l}$ ) on the boundary of $\Omega$ of smallest (resp. largest) abscissa (hence independant of $z$ ). The segment $\left(z_{l}, z\right)$ may be replaced with any path from $\omega_{l}$ to $z$. In case of an even coefficient $\alpha$, another path from $\omega_{r}$ to $z$ can be fixed to obtain a path from $\omega_{r}$ to $\omega_{l}$ that separates $\Omega$ into a lower and an upper part. 


\section{The Dirichlet-Neumann map for the case of an even coefficient $\alpha$}

This section is devoted to the proof of Theorem 1.5. Theorems 1.1 and 1.3 give an expression of the solution to (1.5), and in particular of the jump $J(z, k)$, in terms of its tangential and normal derivatives on the boundary of the disk $\mathcal{D}_{a}$. In practice, only one type of boundary data is usually known and it is thus important to determine if this information is sufficient for computing the solution.

Using the link (3.7) between complex and directional derivatives, the global relation, derived from Proposition 2.1 and Poincaré lemma,

$$
\int_{\mathcal{C}_{a}}[(k-z)(k+\bar{z})]^{\alpha / 2-1}\left((k+\bar{z}) u_{z}(z) d z+(k-z) u_{\bar{z}}(z) d \bar{z}\right)=0,
$$

can be rewritten as

$$
\int_{\mathcal{C}_{a}}[(k-z)(k+\bar{z})]^{\alpha / 2-1}\left((y+i k) u_{t}(z)-x u_{n}(z)\right) d s=0,
$$

for $k \in \mathbb{C} \backslash\left(\mathcal{D}_{a} \cup \mathcal{D}_{-a}\right)$. We first show that, when $\alpha$ is an even negative integer, (4.1) allows one to recover the Neumann data $u_{n}$ from the Dirichlet data $u_{t}$.

\subsection{Case of an even negative integer coefficient $\alpha$}

Proof of Theorem 1.5 when $\alpha=-2(m-1), m \in \mathbb{N}^{*}$. Equivalently to recovering the Neumann data $u_{n}$, we may recover the function

$$
f(z):=(x+a) u_{n}(z+a), \quad z \in \mathbb{T},
$$

from the relation

$$
\int_{\mathbb{T}} \frac{z^{m-1} f(z)}{(z-(k-a))^{m}\left(z+(k+a)^{-1}\right)^{m}} d z=\int_{\mathbb{T}} \frac{z^{m-1}(y+i k) u_{t}(z+a)}{(z-(k-a))^{m}\left(z+(k+a)^{-1}\right)^{m}} d z,
$$

where $k \in \mathbb{C} \backslash\left(\mathcal{D}_{a} \cup \mathcal{D}_{-a}\right)$ and we integrate on $\mathbb{T}$ instead of $\mathcal{C}_{a}$.

Now, we set

$$
\mu:=-1 /(k+a), \quad \varphi(z):=\frac{-z}{1+2 a z} .
$$

The map $\varphi$ is involutive and sends $\mu$ to $1 /(k-a)$. Moreover, $k \in \mathbb{C} \backslash\left(\mathcal{D}_{a} \cup \mathcal{D}_{-a}\right)$ is equivalent to the fact that $\mu$ (or $\varphi(\mu))$ belongs to the annulus

$$
\mathbb{A}:=\mathbb{D} \backslash D\left(\frac{-2 a}{4 a^{2}-1}, \frac{1}{4 a^{2}-1}\right)
$$

so that (4.3) can be rewritten

$$
\int_{\mathbb{T}} \frac{z^{m-1} f(z)}{(1-\varphi(\mu) z)^{m}(z-\mu)^{m}} d z=\frac{1}{\mu} \int_{\mathbb{T}} \frac{z^{m-1}(\mu y-i(a \mu+1)) u_{t}(z+a)}{(1-\varphi(\mu) z)^{m}(z-\mu)^{m}} d z, \quad \mu \in \mathbb{A} .
$$


The functions $f(z), u_{t}(z+a), y u_{t}(z+a)$ are real-valued in $L^{2}(\mathbb{T})$, so they can be decomposed as

$$
f(z)=g_{1}(z)+\bar{g}_{1}(1 / z), \quad u_{t}(z+a)=g_{2}(z)+\bar{g}_{2}(1 / z), \quad y u_{t}(z+a)=g_{3}(z)+\bar{g}_{3}(1 / z),
$$

with $g_{i}(z)$ holomorphic on $\mathbb{D}$ and $\bar{g}_{i}(1 / z)$ holomorphic on $\overline{\mathbb{C}} \backslash \mathbb{D}$ for $i=1,2,3$. Note that the imaginary parts of $g_{1}(0), g_{2}(0), g_{3}(0)$ can be chosen at will in the decompositions (4.5). For $i=1,2,3$, let $h_{i}(z):=z^{m-1} g_{i}(z)$, and

$$
\Phi_{i}(\mu):=\int_{\mathbb{T}} \frac{z^{m-1} g_{i}(z)}{(1-\varphi(\mu) z)^{m}(z-\mu)^{m}} d z=\frac{2 i \pi}{(m-1) !}\left(\frac{h_{i}(z)}{(1-\varphi(\mu) z)^{m}}\right)^{(m-1)}(\mu), \quad \mu \in \mathbb{A} .
$$

It is clear that the functions $\Phi_{i}$ are analytic in $\mathbb{A}$. Since

$$
1-\varphi(\mu) \mu=\left(\mu^{2}+2 a \mu+1\right) /(1+2 a \mu),
$$

one derives from the second expressions in (4.6) that the $\Phi_{i}$ extend analytically to $\mathbb{D}$, except at the point $z_{1}$, where we denote by $z_{1}$ and $z_{2}$ the two roots of $z^{2}+2 a z+1=0$,

$$
z_{1}=-a+\sqrt{a^{2}-1} \in D, \quad z_{2}=\varphi\left(z_{1}\right)=-a-\sqrt{a^{2}-1} \in \mathbb{C} \backslash \mathbb{D} .
$$

At the point $z_{1}$, the functions $\Phi_{i}$ have a polar singularity of order at most $2 m-1$. We also note that the $\Phi_{i}$ have a zero of order at least $m$ at $-1 / 2 a$.

Next, from the fact that $\varphi$ is involutive, follows that, for any function $g(z)$ on $\mathbb{T}$,

$$
\int_{\mathbb{T}} \frac{z^{m-1} \bar{g}(1 / z)}{(1-\varphi(\mu) z)^{m}(z-\mu)^{m}} d z=-\int_{\mathbb{T}} \overline{\frac{z^{m-1} g(z)}{(1-\bar{\mu} z)^{m}(z-\varphi(\bar{\mu}))^{m}} d z .}
$$

Therefore, the relation (4.4) can be rewritten as

$$
\Phi_{1}(\mu)-\bar{\Phi}_{1}(\varphi(\mu))=\Phi_{3}(\mu)-\bar{\Phi}_{3}(\varphi(\mu))-i(a+1 / \mu)\left(\Phi_{2}(\mu)-\bar{\Phi}_{2}(\varphi(\mu))\right), \quad \mu \in \mathbb{A} .
$$

Note that $g_{2}(0)=0$ since the Fourier coefficient of $u_{t}(z+a)$ of order 0 vanishes. In view of (4.6), this entails that $\Phi_{2}(0)=0$ so that the function $\Phi_{2}(\mu) / \mu$ is analytic at 0 . Multiplyimg both sides of the equation by

$$
S(\mu)=\left(\mu-z_{1}\right)^{2 m-1}\left(\mu-z_{2}\right)^{2 m-1} /(2 a \mu+1)^{m},
$$

and rearranging terms, we get

$$
\begin{aligned}
S(\mu)\left(\Phi_{1}(\mu)-\Phi_{3}(\mu)+i(a+1 / \mu)\right. & \left.\Phi_{2}(\mu)\right) \\
& =S(\mu)\left(\bar{\Phi}_{1}(\varphi(\mu))-\bar{\Phi}_{3}(\varphi(\mu))+i(a+1 / \mu) \bar{\Phi}_{2}(\varphi(\mu))\right) .
\end{aligned}
$$

The function on the left-hand side is analytic in $\mathbb{D}$. Moreover, the function $\varphi$ maps $\mathbb{A}$ onto itself and sends the inner disk $D$ onto $\mathbb{C} \backslash \mathbb{D}$ with the inner circle of $\mathbb{A}$ onto the unit circle $\mathbb{T}$. Also, $-1 / 2 a$ and $\infty$ map each others by $\varphi$. Hence, the term on the right-hand side is analytic in $\mathbb{C} \backslash \mathbb{D}$ and has a polar singularity of order $2 m-2$ at infinity. Equating the left-hand side to the part in the right-hand side that is analytic in $\mathbb{D}$, we get

$$
S(\mu) \Phi_{1}(\mu)=S(\mu) \Psi(\mu)+P_{2 m-2}(\mu), \quad \Psi(\mu):=\Phi_{3}(\mu)-i(a+1 / \mu) \Phi_{2}(\mu),
$$


where $\Psi(\mu)$ is a known function depending on $u_{t}$, and $P_{2 m-2}(\mu)$ is a polynomial of degree at most $2 m-2$, that depends on both $u_{t}$ and $u_{n}$, (hence is unknown). Making use of the second expressions in (4.6) of the $\Phi_{i}$ functions and applying Leibniz rule on the $(m-1)$-th derivatives, we readily obtain that $h_{1}(z)$ satisfies the differential equation of order $m-1$,

$$
\sum_{p=0}^{m-1} \alpha_{p} z^{m-1-p}\left(z^{2}+2 a z+1\right)^{p} h_{1}^{(p)}(z)=H(z)+P_{2 m-2}(z),
$$

where

$$
\alpha_{p}=(-1)^{m-1+p} \frac{(2 m-p-2) !}{(m-p-1) ! p !}, \quad p=0, \ldots, m-1,
$$

and $H(z)$ is a known function, analytic in $\mathbb{D}$, that is given in terms of $h_{2}(z)$ and $h_{3}(z)$ by

$$
H(z)=\sum_{p=0}^{m-1} \alpha_{p} z^{m-1-p}\left(z^{2}+2 a z+1\right)^{p}\left(h_{3}^{(p)}(z)-i(a+1 / z) h_{2}^{(p)}(z)\right) .
$$

By Lemma 4.1 which, for convenience, we have stated and proved after the present proof, there exists a unique solution $\widetilde{P}_{2 m-2}(z)$, polynomial of degree at most $2 m-2$, to the differential equation

$$
\sum_{p=0}^{m-1} \alpha_{p} z^{m-1-p}\left(z^{2}+2 a z+1\right)^{p} \widetilde{P}_{2 m-2}^{(p)}(z)=P_{2 m-2}(z) .
$$

Hence, $h_{1}(z)-\widetilde{P}_{2 m-2}(z)$ coincides with the solution $h(z)$, analytic at $z_{1}$, of the differential equation

$$
\sum_{p=0}^{m-1} \alpha_{p} z^{m-1-p}\left(z^{2}+2 a z+1\right)^{p} h^{(p)}(z)=H(z) .
$$

Note that the function $h(z)$ can be explicitely computed as a series expansion around $z_{1}$, see Lemma 4.1. We know that $h_{1}(z)$ vanishes at the origin with order $m-1$, hence it can be written as

$$
h_{1}(z)=z^{m-1}\left(\widetilde{h}(z)+\widetilde{P}_{m-1}(z)\right),
$$

where $\widetilde{h}(z)$ is known and $\widetilde{P}_{m-1}(z)$ is some polynomial of degree at most $m-1$. From the link between $f(z)$ and $h_{1}(z)$ follows that

$$
z^{m-1} f(z)=z^{m-1}(\widetilde{h}(z)+\widetilde{\widetilde{h}}(1 / z))+Q_{2 m-2}(z)
$$

where

$$
Q_{2 m-2}(z)=z^{m-1}\left(\widetilde{P}_{m-1}(z)+\widetilde{\widetilde{P}}_{m-1}(1 / z)\right)
$$

is a polynomial of degree at most $2 m-2$. From the global relation (4.4) and the fact that $\widetilde{h}(z)$ is known, we derive that the following integral is also known for $\mu \in \mathbb{A}$,

$$
\begin{aligned}
\frac{S(\mu)}{2 i \pi} \int_{\mathbb{T}} \frac{Q_{2 m-2}(z)}{(1-\varphi(\mu) z)^{m}(z-\mu)^{m}} d z & =\frac{S(\mu)}{(m-1) !}\left(\frac{Q_{2 m-2}(z)}{(1-\varphi(\mu) z)^{m}}\right)^{(m-1)}(\mu) \\
& =\frac{1}{(m-1) !} \sum_{p=0}^{m-1} \alpha_{p} \mu^{m-1-p}\left(\mu^{2}+2 a \mu+1\right)^{p} Q_{2 m-2}^{(p)}(\mu)
\end{aligned}
$$


Consequently, $Q_{2 m-2}(z)$ solves a differential equation of the type (4.9) with a second member which is necessarily a polynomial, hence analytic at $z_{1}$. From Lemma 4.1, we know that $Q_{2 m-2}$ is uniquely determined and can be recovered from this equation. Therefore, $\widetilde{P}_{m-1}(z), h_{1}(z), g_{1}(z)$, and finally $f(z)$ are also uniquely recovered.

Lemma 4.1. Let $H(z)$ be a function analytic in a neighborhood of $z_{1}$. There exists at most one solution $h(z)$ of the differential equation

$$
\sum_{p=0}^{m-1} \alpha_{p} z^{m-1-p}\left(z^{2}+2 a z+1\right)^{p} h^{(p)}(z)=H(z),
$$

which is analytic in a neighborhood of $z_{1}$. Moreover, if $H(z)=P_{2 m-2}(z)$ is a polynomial of degree at most $2 m-2$, then the solution $h(z)$ exists and is also a polynomial of degree at most $2 m-2$.

Proof. The two roots $z_{1}$ and $z_{2}$ of $z^{2}+2 a z+1$ are regular singular points of the differential equation (4.9). We rewrite it in a neighborhood of $z_{1}=-a+\sqrt{a^{2}-1} \in D$, leading to

$$
\sum_{p=0}^{m-1} \alpha_{p}\left(z+z_{1}\right)^{m-1-p}(z+\beta)^{p} z^{p} \widetilde{h}^{(p)}(z)=H\left(z+z_{1}\right)
$$

where $\beta=2 \sqrt{a^{2}-1}$ and $\widetilde{h}(z)=h\left(z+z_{1}\right)$. Denoting by $\sum_{k=0}^{\infty} a_{k} z^{k}$ the series expansion of $\widetilde{h}$ in a neighborhood of 0 , we have

$$
z^{p} \widetilde{h}^{(p)}(z)=\sum_{k=p}^{\infty} k \ldots(k-p+1) a_{k} z^{k}
$$

and therefore

$$
\sum_{p=0}^{m-1} \alpha_{p}\left(z+z_{1}\right)^{m-1-p}(z+\beta)^{p} \sum_{k=p}^{\infty} k \ldots(k-p+1) a_{k} z^{k}=H\left(z+z_{1}\right) .
$$

We compute the coefficient of $z^{n}$ in the left-hand side of (4.10) which equals

$$
\frac{1}{n !}\left(\sum_{p=0}^{m-1} \alpha_{p}\left(z+z_{1}\right)^{m-1-p}(z+\beta)^{p} \sum_{k=p}^{\infty} a_{k} \frac{k !}{(k-p) !} z^{k}\right)^{(n)}
$$

By Leibniz rule we get

$$
\frac{1}{n !} \sum_{p=0}^{m-1} \alpha_{p} \sum_{k_{1}+k_{2}+k_{3}=n} \frac{1}{k_{1} ! k_{2} ! k_{3} !} \frac{(m-1-p) !}{\left(m-1-p-k_{1}\right) !} z_{1}^{m-1-p-k_{1}} \frac{p !}{\left(p-k_{2}\right) !} \beta^{p-k_{2}} a_{k_{3}} \frac{\left(k_{3} !\right)^{2}}{\left(k_{3}-p\right) !},
$$

where we adopt the convention that $1 / q !=0$ if $q$ is a negative integer. Taking into account the definition of $\alpha_{p}$, this simplifies to

$$
\frac{1}{n !} \sum_{p=0}^{m-1}(-1)^{p}(2 m-p-2) !\left(\frac{\beta}{z_{1}}\right)^{p} \sum_{k_{1}+k_{2}+k_{3}=n} \frac{k_{3} ! z_{1}^{-k_{1}} \beta^{-k_{2}}}{k_{1} ! k_{2} !\left(m-1-p-k_{1}\right) !\left(p-k_{2}\right) !\left(k_{3}-p\right) !} a_{k_{3}} .
$$


Let us write $k_{3}=n-\ell$ where $\ell$ is a new index. Then $k_{1}+k_{2}=\ell$ in the previous sum. Since $k_{1} \leq m-p-1$ and $k_{2} \leq p$, we deduce that $\ell$ takes its values in the set $\{0, \ldots, m-1\}$. We thus obtain

$$
\frac{1}{n !} \sum_{\ell=0}^{m-1}(n-\ell) ! a_{n-\ell} \sum_{p=0}^{m-1} \frac{(-1)^{p}(2 m-p-2) !}{(n-\ell-p) !}\left(\frac{\beta}{z_{1}}\right)^{p} \sum_{k_{1}+k_{2}=\ell} \frac{z_{1}^{-k_{1}} \beta^{-k_{2}}}{k_{1} ! k_{2} !\left(m-1-p-k_{1}\right) !\left(p-k_{2}\right) !},
$$

or writing $k_{1}=k-p$ and $k_{2}=p+\ell-k$ in terms of a new index $k$,

$$
\frac{1}{n !} \sum_{\ell=0}^{m-1} a_{n-\ell} \frac{(n-\ell) !}{\beta^{\ell}} \sum_{p=0}^{m-1}(-1)^{p} \frac{(2 m-p-2) !}{(n-\ell-p) !} \sum_{k=0}^{m-1} \frac{\left(\beta / z_{1}\right)^{k}}{(k-p) !(\ell+p-k) !(m-1-k) !(k-\ell) !} .
$$

Interchanging the two last sums, we obtain

$$
\sum_{\ell=0}^{m-1} a_{n-\ell} \beta_{\ell}^{n}, \quad n \geq 0
$$

where $a_{n}=0, n<0$, and we have set, for $\ell \in\{0, \ldots, m-1\}$,

$$
\beta_{\ell}^{n}:=\frac{(n-\ell) !}{n ! \beta^{\ell}} \sum_{k=0}^{m-1} \frac{\left(\beta / z_{1}\right)^{k}}{(m-1-k) !(k-\ell) !} \sum_{p=0}^{m-1} \frac{(-1)^{p}(2 m-p-2) !}{(k-p) !(n-\ell-p) !(\ell+p-k) !} .
$$

If $\ell=0$, the second sum in (4.11) equals

$$
\sum_{p=0}^{m-1} \frac{(-1)^{p}(2 m-p-2) !}{(k-p) !(n-p) !(p-k) !}=(-1)^{k} \frac{(2 m-k-2) !}{(n-k) !}
$$

and

$$
\beta_{0}^{n}=\sum_{k=0}^{m-1} \frac{\left(-\beta / z_{1}\right)^{k}(2 m-k-2) !}{(m-1-k) ! k !(n-k) !} \neq 0,
$$

since $\beta / z_{1}<0$. The nonvanishing of the coefficients $\beta_{0}^{n}$ implies that each coefficient $a_{n}$, $n>0$, is uniquely determined whence the first assertion of the lemma.

Next, in order to prove the vanishing of all coefficients $a_{n}, n \geq 2 m-1$, when the right-hand side is a polynomial of degree at most $2 m-2$, it is sufficient to show that

$$
\beta_{N+1}^{n}=\cdots=\beta_{m-1}^{n}=0, \quad N \in\{0 \ldots, m-2\}, \quad n=2 m-1+N .
$$

Let $N$ be fixed in $\{0 \ldots, m-2\}$ and $\ell \in\{N+1, \ldots, m-1\}$. From our convention on the factorials of negative integers, the second sum in (4.11) actually simplifies to

$$
\begin{aligned}
\sum_{p=k-\ell}^{k} \frac{(-1)^{p}(2 m-p-2) !}{(k-p) !(n-\ell-p) !(\ell+p-k) !} & =(-1)^{k-\ell} \sum_{p=0}^{\ell} \frac{(-1)^{p}(2 m-k+\ell-p-2) !}{(\ell-p) ! p !(n-k-p) !} \\
& =(-1)^{k-\ell} \sum_{p=0}^{\ell} \frac{(2 m-k+\ell-p-2) !}{(2 m-1+N-k-p) !} \frac{(-1)^{p}}{p !(\ell-p) !}
\end{aligned}
$$


The last expression equals the derivative of order $\ell-(N+1)$ of the polynomial

$$
(-1)^{N+1-\ell} \sum_{p=0}^{\ell} \frac{x^{2 m-k+\ell-p-2}}{p !(\ell-p) !}
$$

taken at $x=-1$. Thanks to the binomial identity, this polynomial rewrites as

$$
(-1)^{N+1-\ell} x^{2 m-k-2}(1+x)^{\ell} / \ell !
$$

We then get

$$
\sum_{p=0}^{m-1} \frac{(-1)^{p}(2 m-p-2) !}{(k-p) !(n-\ell-p) !(\ell+p-k) !}=0
$$

which shows (4.12).

\subsection{Case of an even positive integer coefficient $\alpha$}

Here we assume $\alpha=2(m+1), m \in \mathbb{N}$. First, let us check that the global relation (4.1) does not allow the reconstruction of $u_{n}$ in this case. Indeed, integrating on $\mathbb{T}$ instead of $\mathcal{C}_{a}$, the part involving $u_{n}$ in (4.1) now equals

$$
\int_{\mathbb{T}} \frac{(z-(k-a))^{m}(z+1 /(k+a))^{m} f(z)}{z^{m+1}} d z
$$

where $f$ is defined by $(4.2)$ and $k \in \mathbb{C} \backslash\left(\mathcal{D}_{a} \cup \mathcal{D}_{-a}\right)$. With the definitions of $g_{1}, \mu, \varphi$ and $\mathbb{A}$ as in the previous case, we let, for $\mu \in \mathbb{A}$,

$$
\Phi_{1}(\mu):=\int_{\mathbb{T}} \frac{(z-\mu)^{m}(1-\varphi(\mu) z)^{m} g_{1}(z)}{z^{m+1}} d z=\frac{2 i \pi}{m !}\left((z-\mu)^{m}(1-\varphi(\mu) z)^{m} g_{1}(z)\right)^{(m)}(0),
$$

so that, as before, (4.13) can be rewritten as $\Phi_{1}(\mu)-\bar{\Phi}_{1}(\varphi(\mu))$. Since this expression only involves the derivatives of $g_{1}$ at 0 of order up to $m$, it does not contain enough information to reconstruct $g_{1}(z)$ and a fortiori the function $f(z)$.

Proof of Theorem 1.5 when $\alpha=2(m+1), m \in \mathbb{N}$. Expressing the closed differential form (2.6) in terms of the directional derivatives, thanks to (3.7), and integrating along $\mathcal{C}_{a}$, we now obtain

$$
\int_{\mathcal{C}_{a}} \frac{\left((y+i k) x u_{t}(z)-x^{2} u_{n}(z)-(2 m+1)(z \bar{z}+i y k) u(z)\right) x^{2 m}}{(k-z)^{m+1}(k+\bar{z})^{m+1}} d s=0,
$$

for $k \in \mathbb{C} \backslash\left(\mathcal{D}_{a} \cup \mathcal{D}_{-a}\right)$. Since we assume that $u$ and $u_{t}$ are known on the boundary $\mathcal{C}_{a}$ of the domain, our problem is to recover $u_{n}$ from the knowledge, for $k \in \mathbb{C} \backslash\left(\mathcal{D}_{a} \cup \mathcal{D}_{-a}\right)$, of the integral

$$
\int_{\mathcal{C}_{a}} \frac{x^{2 m+2} u_{n}(z)}{(k-z)^{m+1}(k+\bar{z})^{m+1}} d s,
$$

which is precisely what was done in the previous case (except that the definition (4.2) of the function $f$ there has to be replaced with $\left.f(z)=(x+a)^{2 m+2} u_{n}(z+a)\right)$. 


\section{References}

[1] M. J. Ablowitz and A. S. Fokas, Complex Variables: Introduction and Applications, 2nd Edition, Cambridge University Press, Cambridge, UK (2003).

[2] F. Alladio and F. Crisanti, Analysis of MHD equilibria by toroidal multipolar expansions, Nuclear Fusion, 26 (1986), 1143-1164.

[3] K. Astala and L. Päivärinta, Calderón's inverse conductivity problem in the plane. Ann. of Math. (2), 163 (2006), 265-299.

[4] L. Baratchart, J. Leblond, S. Rigat, E. Russ, Hardy spaces for the conjugate Beltrami equation in smooth domains of the complex plane, J. Funct. Anal. 259, (2010) 384-427.

[5] J. Blum, Numerical simulation and optimal control in plasma physics: with applications to Tokamaks, Modern Applied Mathematics, John Wiley and Sons (1989).

[6] S. Chaabi, Analyse complexe et problèmes de Dirichlet dans le plan : équation de Weinstein et autres conductivités non-bornées. PhD thesis, Aix-Marseille université, 2013.

[7] S. Chaabi, S. Rigat, Decomposition theorem and Riesz basis for axisymmetric potentials in the right half-plane, Arxiv 1402.0473.

[8] A.S. Fokas, A unified approach to boundary value problems, CBMS-NSF Regional Conference Series in Applied Mathematics, 78, SIAM, Philadelphia, PA, 2008.

[9] G. B. Folland, Introduction to partial differential equations, Princeton University press, Princeton, N. J., 1975.

[10] F.D. Gakhov, Boundary Value Problems, Dover Publications, New York 1990.

[11] D. Gilbarg, N. Trudinger, Elliptic Partial Differential Equations of Second Order, Springer Verlag, New York, 1983.

[12] R.P. Gilbert, On generalized axially symmetric potentials, J. Reine Angew. Math. 212 (1963) 158-168.

[13] R.P. Gilbert, Some properties of generalized axially symmetric potentials, Amer. J. Math. 84 (1962) 475-484.

[14] R.P. Gilbert, Bergman's integral operator method in generalized axially symmetric potential theory, J. Math. Physics 5 (1964) 983-997.

[15] R.P. Gilbert, Poisson's equation and generalized axially symmetric potential theory, Ann. Mat. Pura Appl. 61 (1963) 337-348.

[16] P. Henrici, On the domain of regularity of generalized axially symmetric potentials, Proc. Amer. Math. Soc. 8 (1957) 29-31. 
[17] P. Henrici, A survey of I. N. Vekua's theory of elliptic partial differential equations with analytic coefficients, Z. Angew. Math. Phys. 8 (1957) 169-203.

[18] P. Henrici, Complete systems of solutions for a class of singular elliptic partial differential equations, Boundary problems in differential equations, 19-34, Univ. of Wisconsin Press, Madison, 1960.

[19] N.N. Lebedev, Special functions and their applications, Dover, 1972.

[20] J. Lenells, A.S. Fokas, Boundary-value problems for the stationary axisymmetric Einstein equations: a rotating disc, Nonlinearity 24 (2011), 177-206.

[21] A. Mackie, Contour integral solutions of a class of differential equations, J. Rat. Mech. Anal. 4 (1955) 733-50.

[22] P.M. Morse, H. Feshbach, Methods of Theoretical Physics. Cambridge University press, 1953.

[23] K. Ranger, Some integral transformation formulae for the Stokes-Beltrami equations, J. Math. Mech. 12 (1963) 663-673.

[24] V.D. Shafranov, On magnetohydrodynamical equilibrium configurations, Soviet Physics JETP, 6 (1958), 545-554.

[25] A. Tovbis and S. Venakides, Semiclassical Limit of the Scattering Transform for the Focusing Nonlinear Schrödinger Equation, Int. Math. Res. Notices 2012 (2012), 22122271.

[26] B. Ph. van Milligen, A. Lopez Fraguas, Expansion of vacuum magnetic fields in toroidal harmonics, Computer Physics Communications 81 (1994), 74-90.

[27] I.N. Vekua, New methods for solving elliptic equations, John Wiley \& Sons, New York 1967.

[28] A. Weinstein, Transonic flow and generalized axially symmetric potential theory, Symposium on theoretical compressible flow, Naval Ordnance Laboratory, White Oak, Md., (1950), 73-82.

[29] A. Weinstein, Generalized axially symmetric potential theory, Bull. Amer. Math. Soc. 59, (1953), 20-38.

[30] A. Weinstein, On a class of partial differential equations of even order, Ann. Mat. Pura Appl. 39 (1955), 245-254.

[31] A. Weinstein, The method of axial symmetry in partial differential equations, Convegno Internazionale sulle Equazioni Lineari alle Derivate Parziali, Trieste, 1954, 8696. Edizioni Cremonese, Roma, 1955. 
Slah Chaabi, Stephane Rigat, Franck Wielonsky

slah.chaabi, stephane.rigat, franck.wielonsky@univ-amu.fr

Laboratoire I2M - UMR CNRS 7373

Université Aix-Marseille

CMI 39 Rue Joliot Curie

F-13453 Marseille Cedex 20, FRANCE 